\title{
AN OVERVIEW ON TRICHOMONAS VAGINALIS WITH REFERENCE TO EGYPT \\ By
}

TAREK ABDEL KADER SALLAM ${ }^{1 *}$, LAILA ABDEL-MAWLA MEGAHED ${ }^{1}$, SHEREEN MOHAMAD IBRAHIM ${ }^{1}$ and TOSSON A. MORSY ${ }^{2}$

Military Medical Academy, Cairo, $11291^{1}$, Egypt and Department of Parasitology, Faculty of Medicine, Ain Shams University, Cairo $11566^{2}$, Egypt

( ${ }^{*}$ Correspondence: ${ }^{*}$ trksaam35@gmail.com, **tossonmorsy@ med.asu.edu.eg or morsyegypt2014@gmail.comorcid.org/0000-0003-2799-2049)

Abstract

Trichomoniasis is the most prevalent non-viral sexually transmitted infection worldwide. It is associated with a high prevalence of coinfection with other sexually transmitted diseases and can be identified in 30 to $40 \%$ of male sexual partners of infected women. Some possible consequences of trichomonaisis in women include pelvic inflammatory disease, post-hysterectomy cellulitis, and preterm birth; some possible consequences in men include prostatitis, infertility, and prostate cancer. Trichomonas infection in women appears to increase the risk of sexual acquisition of the HIV, preterm birth, and other adverse pregnancy outcomes

Clinical manifestations in women range from an asymptomatic carrier state to a severe, acute, inflammatory disease. Signs and symptoms include a purulent, malodorous, thin discharge with associated burning, pruritus, dysuria, frequency, and dyspareunia.

Key words: Trichomonas vaginalis, signs, symptoms, treatment, risky complications

\section{Introduction}

Trichomonas vaginalis is the causative agent of trichomoniasis. It is the most common non-viral sexually transmitted infection worldwide and accounts for 4 to $35 \%$ of vaginitis diagnosed in symptomatic women presenting in primary care settings in the United States (Anderson et al, 2004).

Prevalence: The prevalence of trichomonas vaginalis infection in reproductive aged women in the US was illustrated by a study in which 3754 women aged 14 to 49 years self-collected vaginal swab specimens that were subsequently evaluated for the presence of $T$. vaginalis using PCR (Sutton et al, 2007). The overall prevalence of $T$. vaginalis was $3.1 \%$, and increased with age. Prevalence was highest in non-Hispanic black women $(13.3 \%)$ and lowest in non-Hispanic white women $(1.3 \%)$. The annual incidence in the United States was estimated to be three to five million cases (Ginocchio et al, 2012).

\section{Review and Discussion}

Microbiology and risk factors: The responnsible organism is the flagellated protozoan trichomonas vaginalis, which may be found in the vagina, urethra, and paraurethral gla- nds of infected women. Other sites include cervix, bladder, and Bartholin glands. Humans are the only natural host of $T$. vaginalis. The incubation period is unknown; but, in vitro studies suggest an incubation period of 4 to 28 days (Hesseltine, 1942)

Although survival on fomites has been reported, trichomoniasis is virtually always sexually transmitted. Fomites have no proven role in transmission of trichomonas infection. Trichomoniasis is associated with a high prevalence of coinfection with other sexually transmitted diseases. The organism can be identified in 30 to $40 \%$ of the male sexual partners of infected women, although carriage in men is self-limited and transient. Spermicidal agents such as nonoxynol-9 reduce the rate of transmission of trichomonas (d'Oro et al, 1994).

Women can acquire the disease from other women, but men do not usually transmit the infection to other men. Trichomoniasis in women ranges from an asymptomatic carrier state to a severe, acute, inflammatory disease. More than $50 \%$ of infected women are asymptomatic. Asymptomatic carriage can occur for prolonged periods of time, thus it was not necessarily possible to ascertain 
when or from whom acquired the infection (Cudmore et al, 2004). Classic signs and symptoms include a purulent, malodorous, thin discharge ( $70 \%$ of cases) with associated burning, pruritus, dysuria, frequency, and dyspareunia. Post-coital bleeding occurred. Urethra was infected in majority of women, particularly during menstruation. Classically described green, frothy, foul-smelling discharge was found less than $10 \%$ of symptomatic women (Heine and McGregor, 1993).

Physical examination often reveals erythema of the vulva and vaginal mucosa; the classic green-yellow frothy discharge is observed in 10 to $30 \%$ of affected women. Punctate hemorrhages may be visible on the vagina and cervix (strawberry cervix, $2 \%$ of cases).

Men clinical features: $T$ vaginalis infection in men is asymptomatic in $75 \%$ of cases and often transient; (spontaneous resolution within 10 days (Seña et al, 2007). Symptoms, when present ( $<10 \%$ of cases $)$, are the same as those for urethritis from any cause and consist of a clear or mucopurulent urethral discharge and/or dysuria (Stukus et al, 2019).

Consequences: Active trichomoniasis is a risk factor for development of post-hysterectomy cellulitis. Untreated vaginal infection may progress to urethritis or cystitis, and appears to facilitate transmission of the human immunodeficiency virus (Laga et al, 1993).

A history of trichomoniasis was associated with an increased risk of future tubal infertility and cervical neoplasia (Soper, 2004). In a study of 696 African women attending a rural sexually transmitted disease clinic, trichomoniasis was associated with an increased risk of clinical pelvic inflammatory disease in HIV-infected women (Moodley et al, 2002).

Pregnant women: The consequences of trichomoniasis are potentially important in pregn-ant women in whom infection was associated with a $30 \%$ increase in premature rupture of the membranes and preterm de- livery (Cotch et al, 1997). Treatment of asymptomatic infection does not reduce these complications (Klebanoff et al, 2001).

Infants born to infected mothers may contract infection during delivery. Signs and symptoms in neonates may include fever, respiratory problems, urinary tract infection, nasal discharge, and, in girls, vaginal discharge (Hoffman et al, 2003). Treatment of asymptomatic infants was not necessary as spontaneous resolution wouldoccur when estrogen levels wane to normal prepubescent levels (Carter and Whithaus, 2008). Apart from trichomoniasis, vulvovaginitis is one of the commonest gynecological complaints presenting in the pediatric and adolescent female. When a child was presented with vulvar itching, burning and irritation the most common etiology is non-specific and hygiene measures are recommended. However, these symptoms can mimic more serious etiologies including infection, labial adhesion, lichen sclerosis, pinworms and foreign body must be considered. Yeast and bacterial vaginosis are commonly seen but due to the higher rate of sexual activity in this population sexually transmitted infections must also be considered (Loveless and Myint, 2018).

Men: Potential trichomoniasis vaginalis complications include prostatitis (Kim et al, 2017), balanoposthitis (Michalowski, 1981), epididymitis (Sivaraj et al, 2021), infertility (Mielczarek and Blaszkowska, 2016), and prostate cancer. Trichomonas vaginalis was proposed to contribute to the development of chronic prostate conditions, including benign prostatic hyperplasia and prostate cancer (Shui et al, 2016). But, Langston et al. (2019) didn't provide strong support for prostate involvement during $T$. vaginalis infection, and added that positive data for higher PSA concentrations do not rule out possibility entirely, and relevant for prostate condition development because higher early- to mid-life PSA concentrations were predict greater prostate cancer risk later in life.

Diagnosis: Trichomonas diagnosis is based 
on laboratory testing, as described below. As with other types of vaginitis, none of the clinical features of trichomonas vaginitis was sufficiently sensitive or specific to allow a diagnosis based upon signs and symptoms alone (Hammill, 1989). The general diagnostic approach to women with vaginal discharge is given below.

Microscopy and $\mathrm{pH}$ : The presence of motile trichomonads on wet mount is diagnostic of infection, but this occurs in only 60 to $70 \%$ of culture-confirmed cases. The motion is jerky and spinning; organisms remain motile for 10 to 20 minutes after collection of the sample. Other findings that were almost invariably present with trichomonas infection, but non-diagnostic, include an elevated vaginal $\mathrm{pH}(>4.5)$ and an increase in polymorphonuclear leukocytes on saline microscopy (Wølner-Hanssen et al, 1989).

Culture: Culture on Diamond's medium has a high sensitivity (95\%) and specificity $(>95 \%)$ and should be considered in patients with elevated vaginal $\mathrm{pH}$ (Mahmoud et al, 2015), increased numbers of polymorphonuclear leukocytes and an absence of motile trichomonads and clue cells on wet mount; or when microscopy is unavailable or yields unreliable results. This test is not readily available and takes up to seven days to obtain a result. Alternatively, a commercial "In Pouch" T. vaginalis culture system takes up to three days to obtain results and is readily available (Bio-Med Diagnostics, White City, Oregon).

Rapid antigen \& nucleic acid amplification tests: Rapid diagnostic kits can be useful in areas of high prevalence where microscopy or culture is not available (i.e., sexually transmit-ted disease (STD) clinics and inner city clinics serving the uninsured were high prevalence sites). The following tests are performed at the point of care and are commercially available (Smith et al, 2002).

1- APTIMA Trichomonas vaginalis assay (Gen-Probe): The APTIMA T. vaginalis assay uses target capture and transcription mediated amplification to detect species specif- ic ribonucleic acid (RNA), including $N$. gonorrhoeae, $C$. trachomatis and $T$ vaginalis on a vaginal swab or urine specimen (Krieger et al, 1988). Reported sensitivity ranges from 97 to $100 \%$ and specificity ranges from 87 to $100 \%$ (Chapin and Andrea, 2011). The APTIMA TV assay showed superior performance in side-by-side comparisons with other diagnostic methods in all patient populations and specimen types tested as vaginal discharge, \& urine (McClelland et al, 2007). 2- AFFIRM VP III - The Affirm VP III Microbial Identification System (Becton Dickins-on) test uses a nucleic acid probe on a vaginal swab specimen. Results are available in 45 minutes. Although sensitivity and specificity were reported to exceed $95 \%$, in side-by-side testing, this method was inferior to target capture and transcription mediated amplification with sensitivity 63.4 versus 100\% (Schwebke et al, 2011).

3- OSOM - The OSOM Trichomonas Rapid Test (Genzyme) uses an immunochromatographic technology on a vaginal swab specimen. Results are available in 10 minutes. It has sensitivity of $88.3 \%$ and specificity of 98.8\% (Huppert et al, 2005).

The excellent performance characteristics of these tests were illustrated by the following comparative studies, which compare performance of traditional and rapid testing methods: One study recruited 330 sexually active adolescent females seeking care at a teen health clinic or emergency department and tested them for $T$. vaginalis using the four test methods discussed: wet mount, culture (InPouch TV), rapid antigen testing (OSOM TV), and transcription-mediated amplification testing (APTIMA TV). Sensitivities of the four methods were $65,96,90$, \& 98\%, respectively (Huppert et al, 2007). Another study enrolled 296 female and 298 male subjects seeking care at a sexually transmitted disease clinic. Specimens from each subject were tested for trichomoniasis using transcription-mediated amplification (APTIMA TV), wet mount microscopy, culture, and PCR tests (Nye et al, 2009). The 
subject was diagnosed with trichomoniasis if any of these tests was positive. In women, vaginal swab APTIMA TV was more sensitive than wet mount or culture (96.6 versus $54.6 \& 75.0 \%$, respectively). In men, urethral swab APTIMA TV was more sensitive than culture or PCR (95.2 versus $28.6 \&$ $54.8 \%$, respectively (Grodstein et al, 1993). In a third study, specimens were retrieved consecutively from 766patients with vaginal complaints and/or with histories suggestive of a sexually transmitted disease and tested by the AFFIRM and APTIMA T. vaginalis assays (Andrea and Chapin, 2011). Overall, $5.1 \%$ of patients were positive for $T$ vaginal$i s$ (defined as a specimen with two positive test results for $T$. vaginalis by two different molecular assays). The APTIMA assay was statistically more sensitive than the AFFIRM assay (100 versus 63.4\%); specificity was similar (APTIMA 100\%, AFFIRM 99.9\%).

Amplicor (Roche) is a PCR assay for detection of $\mathrm{N}$ gonorrhoeae and $\mathrm{C}$ trachomatis that has been modified to detect $T$ vaginalis in vaginal/endocervical swabs or urine; sensitivity and specificity are 88 to $97 \%$ and 98 to $99 \%$, respectively (Van Der Pol et al, 2006). It was not commercially available, but was cleared by the United States Food and Drug Administration (FDA). NuSwab $\mathrm{VG}$ is a nucleic acid amplification single specimen assay for the detection of trichomoniasis, bacterial vaginosis and vulvovaginal candidiasis that has also been cleared by the FDA, but not yet commercially available (Zhang and Begg, 1994).

Cervical cytology: Trichomonas are sometimes reported as an incidental finding on conventional Papanicolaou (Pap) smears. Conventional Pap smears are inadequate for diagnosis of trichomoniasis because this technique gave a sensitivity of only 51 to $63 \%$ $\&$ false positive results were common (7\%). This makes the positive predictive value very poor in a low prevalence population (Wiese et al, 2000). Thus, Pap smear should not be used to diagnose trichomoniasis. Asymptomatic women with trichomonads iden- tified on conventional Pap smear should be evaluated by wet mount, or culture or rapid antigen test if the wet mount was negative, and should not be treated until the diagnosis was confirmed (Temesvári et al, 2002).

By comparison, the reliability of liquidbased cervical cytology appears to be higher. One study performed both liquid-based cervical cytology and culture for $T$. vaginalis on 203 consecutive women. Forty-four women had a positive culture and 28 women had a positive liquid-based smear for trichomonads (Lara-Torre and Pinkerton, 2003). Although the sensitivity of liquid-based smears for Trichomonas infection was low (61\%), specificity was high $(99 \%)$, resulting in a false positive rate of only $1 \%$. Thus, although liquid-based cervical cytology is not a sensitive test for diagnosis of trichomoniasis, treatment of asymptomatic women with trichomonads noted on liquid-based cervical cytology is a reasonable approach.

Men: The reliable test for trichomonas is PCR testing of first fraction urine or a urethral swab, which is not widely available (El-Shazly et al, 2001) and latex agglutination test (Adu-Sarkodie et al, 2004). Other tests, such as saline microscopy of a urethral swab, have low sensitivity and not recommended (Sorvillo and Kerndt, 1998). But, Stoner et al. (2013) in USA reported that vaginal fluid samples for trichomoniasis diagnosis must be stored in saline rather than on microscope slides until they were examined under the microscope and samples should be evaluated by microscopy within an hour of collection. They added that clinical sites that couldnot perform microscopy within an hour of sample collection should consider for use of other diagnostic tests. Bhesania and Narayankhedkar (2016) in India reported that the wet mount tests gave variable, with sensitivities of 38 to $82 \%$ am-ong symptomatic women. Schwebke et al. (2013) in USA despite these statistics the wet mount test is still used in clinical trials for evaluation of drug activity, and must be done within a few minutes after collection for best result of the 
viable parasites.

Screening: There were about 58 million cases of trichomoniasis in 2013 (Vos et al, 2015). Vos et al. (2012) reported that infection was more common in women $(2.7 \%)$ than males $(1.4 \%)$, and was the most common non-viral STI in the U.S., with an estimated 3.7 million prevalent cases and 1.1 million new cases a year (CDC, 2012). Evidence from a randomized controlled trial (McGregor et al, 1995) for screening pregnant women who did not have symptoms for trichomoniasis infection and treating women who test positive for the infection have not consistently shown a reduced risk of preterm birth (Klebanoff et al, 2001). Studies are needed to verify this result and determine the best method of screening, in the US, screening of pregnant women without any symptoms was only recommended in those with HIV as trichomonas infection is associated with increased risk of HIV transmission to the fetus (Workowski and Bolan, 2015). Rowley et al. (2019) mentioned that the WHO (2016) estimated 156 million cases of $T$. vaginalis worldwide in 2016, accounting for almost half of the global STI incidence that year Patel et al. (2018) Updated epidemiological data on the national prevalence of trichomonas among women and men in the U.S. until both partners have waited at least seven days since taking the last antibiotic dose.

Treatment: Patients should be instructed to avoid intercourse until they and their partners have completed treatment and are asymptomatic, which generally takes about a week. After single dose therapy or treatment of asymptomatic patients, the couple should abstain from intercourse without studies on how long trichomonads remain viable after treatment is initiated or completed (Manorama and Shenoy, 1978). However, persistent or recurrent infection due to antimicrobial-resistant $T$. vaginalis or other causes must be differentiated from reinfection from an untreated or insufficiently treated sexual partner. After treatment failure, and if rein- fection was excluded, persistent or recurrent trichomoniasis was treated successfully with longer courses or additional doses of the same medications used in standard therapy as high-dose oral MTZ or TIN $2 \mathrm{gm}$ orally daily for 7 days (Dessì et al, 2019)

Non-pregnant women: Treatment is indicated in all non-pregnant women diagnosed with trichomonas vaginitis, even if asymptomatic. The treatment rationale for asymptomatic women is that if left untreated, up to one-third become symptomatic within six months and they continue to transmit the infection to sexual partners while untreated (Schwebke and Burgess, 2004).

5-nitroimidazole drugs: The 5-nitroimidazole drugs (metronidazole or tinidazole) are the only class of drugs that provide curative therapy of trichomoniasis. Most strains of $T$. vaginalis are highly susceptible to these agents. Randomized clinical trials of these drugs have generally reported cure rates of 90 to $95 \%$ (Hager et al, 1980). Therapy with drugs other than 5-nitroimidazoles is an option, but cure rates were low as $\leq 50 \%$ (Forna and Gülmezoglu, 2003)

A single oral dose of 2 grams (four $500 \mathrm{mg}$ tablets) of either tinidazole or metronidazole, tinidazole was significantly more effective than metronidazole in several randomized trials; however, most of these trials were subject to bias since outcome was assessed by clinicians who were not blinded to the patient's treatment group. Cure rate with tinidazole was considered comparable to the metronidazole, but with better tolerated (Tidwell et al, 1994).

The alternative multi-dose regimen was metronidazole $500 \mathrm{mg}$ orally twice a day for seven days, without FDA approved multiple dose regimen; fortinidazole. Similar cure rates were obtained with single and multiple dose regimens (cure rate for single dose: 82 to $88 \%$; cure rate for multiple dose: 85 to $90 \%$ ). The advantages of single-dose therapy included better compliance, a shorter period of alcohol avoidance, and possibly decreased Candida super-infection (but with- 
out evidence of decreased Candida superinfection). But, side effects (e.g., nausea, vomiting, headache, metallic taste, dizziness) were dose related and occurred less frequently with lower doses of multiple dose; prolonged therapy (duBouchet et al, 1997).

Oral was preferred to vaginal therapy since systemic administration achieves therapeutic drug levels in the urethra and periurethral glands, which served as endogenous reservoirs of organisms that can cause recurrence. Cure rates for vaginal therapy were $\leq 50 \%$, which was significantly lower than with oral therapy. Patients should be advised to not consume alcohol for 24 hours after metronidazole treatment and 72 hours after tinidazole treatment because of the possibility of a disulfiram-like (Antabuse effect) reaction (Thin et al, 1979).

Allergy to 5-nitroimidazole drugs: Given the low efficacy of any drug other than 5-nitroimidazole drugs, and that patients with allergy to metronidazole or tinidazole must be referred for desensitization rather than using an alternative class of drugs. Desensitization was very effective: in one series, all 15 women who underwent desensitization subsequently eradicated their infections (Helms et al, 2008).

Follow-up is unnecessary for women who become asymptomatic after treatment or who were initially asymptomatic, given the high efficacy of 5-nitroimidazole drugs.

Male partners: Treatment of male partners is indicated because maximal cure rates in affected women were achieved when their male sexual partners are treated simultaneously. It is not mandatory to identify the organism in a male partner before treating him (i.e., Expedited Partner Therapy), given the high rate of concurrent carriage (30 to $70 \%$ ), difficulty of diagnosis in males, lower compliance when the partner is asked to visit his health care provider, and the convenience, low morbidity, and low cost of empiric treatment (Lossick, 1990). Antimicrobial treatment of male partners is identical to therapy of non-pregnant females, with preference for single dose regimens to ensure compliance (single oral dose of 2 grams of either tinidazole or metronidazole). Neither partner must resume intercourse until both partners have completed treatment, otherwise reinfection can occur.

Ideally, however, sexual partners should be referred for diagnostic evaluation because concurrent sexually transmitted infections, such as chlamydia and gonorrhea, are common and need to be diagnosed and treated as well, if present. However, if the female diagnosed with chlamydia or gonorrhea, Expedited Partner Therapy (EPT) was also an option for these infections (CDC, 2006a).

Pregnancy: Metronidazole is the drug of choice for treatment of symptomatic trichomoniasis in pregnancy. Some clinicians avoid its use in the first trimester because it crosses the placenta, thus there was a potential for teratogenicity (Kissinger et al, 2006). In addition, the drug is mutagenic in bacteria and carcinogenic in mice. However, metaanalysis has not found any relationship between metronidazole exposure during the first trimester of pregnancy and birth defects (Caro-Patón et al, 1997), but, no longer discourage the use of metronidazole in the first trimester (CDC, 2006).

In pregnant women, some clinicians prefer metronidazole $500 \mathrm{mg}$ twice daily for five to seven days to the $2 \mathrm{~g}$ single dose regimen because of the lower frequency of side effects in women who already have a high prevalence of nausea, but both regimens are acceptable (Muzny and Schwebke, 2013).

Treating asymptomatic infections during pregnancy was recommended because randomized trials have shown that it didn't prevent, and in some trials even increased, the risk of preterm delivery. This was proved in a randomized trial of 617 women with culture proven asymptomatic infection at 16 to 23 weeks of gestation who were randomly assigned to receive either two $2 \mathrm{gm}$ doses of the metronidazole or placebo 48 hours apart (Okun et al, 2005). All women were retreated with the same regimen at 24 to 29 weeks 
of gestation. The major trials were Trichomonas resolved in $93 \%$ of metronidazole treated women and $35 \%$ of the placebo group.

The rate of preterm birth was significantly higher in treated than control women (19 versus $11 \%$, RR 1.8 ; 95\% CI 1.2-2.7), primarily related to an increased frequency of spontaneous preterm labor (10.2 vs. 3.5\%, RR 3.0; 95\% CI 1.5-5.9). The increased risk of preterm delivery was unexpected. Carey and Klebanoff (2005) speculated that use of twice standard single $2 \mathrm{~g}$ metronidazole dose might have disturbed colonization of normal vaginal flora, thus enhancing the potential for preterm labor. Since more extensive culturing was not performed, presence of other infections was not found (Soper et al, 1990).

Asymptomatic women may opt to undergo treatment after 37 weeks to prevent perinatal transmission (CDC, 2010a).

An alternative management of symptomatic infections was clotrimazole $1 \%$ cream inserted vaginally (preferably at bedtime) for seven consecutive days. This often resulted in symptomatic relief, but not eradication of the organisms. There was little information on the safety of tinidazole in pregnancy; therefore, must not suggest its use.

Treatment of sexual partners is indicated. Reinfection of treated partners of untreated women can be minimized by avoidance of sexual intercourse or use of condoms.

Breastfeeding women: When indicated, metronidazole was used for treatment of infection in neonates. There are no human data supporting an association between metronidazole use and cancer; however, an association with carcinogenesis in rodents was demonstrated (Lactmed, 2010). Outcomes data of maternal metronidazole use did not show a significant increase in adverse events compared to use of other antimicrobials, although a cohort study didn't find a significant trend toward more loose stools and more candida colonization in metronidazoleexposed infants. Since the relative infant dose of metronidazole was high $(29 \%)$ with maternal administration of the $2 \mathrm{~g}$ one-time dose, a cautious approach for mothers receiving this dose is to express and discard their milk for 12 to 24 hours to allow excretion of the drug. In mothers using tinidazole, until tinidazole has been proven harmless to the neonate breast feeding after I.V. administration of $1600 \mathrm{mg}$ should not be initiated earlier than 72 hours after the dose (Evaldson et al, 1985).

HIV-positive women: Standard treatment recommendations for trichomoniasis have been based on studies conducted in HIVnegative persons. There is some evidence that HIV-positive women should receive longer treatment. In randomized trial where HIV-positive women with culture confirmed trichomonas vaginalis were treated with metronidazole $2 \mathrm{~g}$ single dose or metronidazole 500mg twice per day for seven days, single dose therapy was less effective than multiple-day therapy (Kissinger et al, 2010). The 7-day treated patients had a lower rate positive cultures 6 to 12 days post-treatment completion $11 / 130$ women $(8.5 \%)$ versus 21/125(16.8\%); RR 0.5, 95\% CI 0.25-1.00) and at 3 months $8 / 73$ women (11\%) versus 19/79(24.1\%); RR 0.46, 95\% CI 0.21-0.98). Of note, all women were given $2 \mathrm{~g}$ metronidazole doses to deliver to their sex partners.

Another study reported a high prevalence of bacterial vaginosis in HIV-positive/Trichomonas-positive women and observed an association between the presence of bacterial vaginosis and early failure of single metronidazole dose to treat trichomonas (Gatski et al, 2011). Although the 2010 CDC STD guidelines did not recommend more prolonged therapy for HIV positive women, it may be prudent to prescribe a seven-day course of therapy, given subsequent publication of additional data on these cases. However, more studies are needed.

Refractory cases: The most common causes of treatment failure are noncompliance and reinfection. Compliance is enhanced with single dose therapy. Reinfection is unlikely if the partner was treated concurrently or 
sexual intercourse was avoided until both partners completed treatment. CDC (2015) recommended for therapy of recurrent trichomoniasis after failure of a single $2 \mathrm{~g}$ dose of metronidazole must be given as $500 \mathrm{mg}$ twice daily for seven days (total dose $7 \mathrm{~g}$ ). If this regimen fails, tinidazole or metronidazole was administered at a dose of $2 \mathrm{~g}$ per day for 5 days (total dose $10 \mathrm{~g}$ ). These regimens were effective in patients with low levels of metronidazole resistance, which was noted in $4 \%$ of $T$. vaginalis isolates of women attending STD clinics in six cities in the United States (Kirkcaldy et al, 2012).

If this fails, the CDC recommends in vitro culture and drug susceptibility testing and referral to a specialist. Therapeutic options include maximum tolerated doses of metronidazole, or preferably tinidazole ( 2 to $4 \mathrm{~g}$ daily in divided doses) for 14 days (Sobel et al, 2001). Considerable success with tinidazole in refractory disease was reported although the optimal dose was not established (Subramanian and Sobel, 2011). Cross resistance to tinidazole was frequent, but not inevitable (Schwebke and Barrientes, 2006). Patients with high level metronidazole resistance were usually successfully treated by prolonged and high dose tinidazole therapy. High dose tinidazole was a better safety profile and was better tolerated than high dose metronidazole (Sobel et al, 1999).

Case reports described successful use of nimorazole, ornidazole, niridazole, furazolidone, and hamycin, whereas a trial of nitazoxanide in three women with difficult to treat $T$. vaginitis reported lack of efficacy (Dan and Sobel, 2007). Rare patients who do not have a response to nitroimidazoles were treated with topical paromomycin as $250 \mathrm{mg}$ daily for two weeks (Nyirjesy et al, 1995). Paromomycin was not available commercially in the United States as a cream and has to be made up by a compounding pharmacy. Little is known about this preparation. Severe local side effects (pain, mucosal ulceration) can occur (Poppe, 2001). Henien et al. (2019) in USA reported that metronidazole- resistant trichomoniasis was not common with significant therapeutic challenges. The combination therapy with high-dose oral tinidazole and vaginal paromomycin cream was uniformly successful. They added that a patient who responded to combination therapy with high-dose oral tinidazole and intravaginal paromomycin.

Prevention: Risk of acquiring infection is reduced by consistent use of condoms and limiting the number of sexual partners, as well as early diagnosis and proper treatment.

Some infected men have symptoms of urethritis, epididymitis, or prostatitis, and some infected women have vaginal discharge that may be diffuse, malodorous, or yellow-green with or without vulvar irritation. But, most infected persons $(70 \%-85 \%)$ have minimal or no symptoms and untreated infections might last for months to years (Peterman et $a l$, 2006). Although partners might be unaware of their infection, it is readily passed between sex partners during penile-vaginal sex (Gray et al, 3009). Among persons who are sexually active, the best way to prevent trichomoniasis is through consistent and correct use of condoms during all penile-vaginal sexual encounters (CDC, 2010b). Partners of men who were circumcised might have a somewhat reduced risk of $T$. vaginalis infection (Tsai et al, 2009). Douching is not recommended because it might increase the risk for vaginal infections, including trichomoniasis (Tsai et al, 2009a).

Whether the rectum can be a reservoir for $T$. vaginalis was unclear; data are needed to clarify whether this occasional result may reflect recent depositing contamination in up to $5 \%$ of persons reporting recent receptive anal sex (Francis al, 2008). Also, efficacy, benefit, and cost-effectiveness of rectal testing are unknown; but, rectal testing for $T$. vaginalis is not recommended. Besides, oral testing for $T$. vaginalis is not recommended because of a lack of evidence for oral infections. Providers should advise persons infected with $T$. vaginalis to abstain from sex until they and their sex partners are treated 
(Sena et al, 2007). Testing for other STDs including HIV should be performed in persons infected with $T$ vaginalis (Tsai et al, $2009 \mathrm{~b}$ ). The etesting for $T$. vaginalis is recommended for all sexually active women within 3 months following initial treatment regardless of whether they believe their sex partners were treated

In Egypt, many authors dealt with $T$. vaginalis, the selected ones in concern were:

Morsy et al. (1984) correlated between clinical and parasitological findings of T. vaginalis among in patient in El Hussein University Gynecological and Obstetrics Hospital.

El Seoud et al. (1988) diagnosed T. vaginalis $28.8 \%$ male patients with urethral discharge and in $8.2 \%$ suffered from impotence and infertility, and Diamond's culture was method of choice. El-Ganayni et al. (1992) found that ELISA sensitivity was $95.7 \%$ \& specificity $91.4 \%$ in contrast to $68.1 \& 77.1 \%$ for counter-immunoelectrrophoresis (CIEP), \& that ELISA could be used for epidemiological study of vaginal trichomoniasis.

Sayed el-Ahl et al. (2002) highlighted the importance of clinically detection of $T$. vaginalis, as one of the group of factors involved in the genesis and progression of cervical cancer. They added that treatment would aid in restricting rising incidence of this dreaded disease. Tawfeek et al. (2003) found that all isolates of $T$. vaginalis release cysteine proteinases proteolytic enzymes that were shed into the vagina or culture medium. Cystatin was used successfully as a capture agent in ELISA to detect cysteine proteinase antibodies without purified proteinases $T$. vaginalis was detected in 19(36.5\%), 49(94.2\%), 50 $(96.1 \%)$ and $48(92.3 \%)$ by wet mount, culture, cystatin capture ELISA-vaginal washouts \& ELISA-sera, respectively. They added that assay was reliable also as a test of cure with a specificity of $94.4 \%$ in the vaginal washouts and $83.3 \%$ in sera. Negm and el-Haleem (2004) found that 21/23 of symptomatic samples were positive for T. vaginalis by one or more methods. Positive by PCR was $21(91.3 \%)$, by culture $17(72.9 \%)$, by
Acridine orange (AO) stain $14(60.8 \%)$ and by wet mount microscopy. 13(56.5\%). PCR detected all positive cases and no PCR negative cases proved to be positive by any other technique. They concluded that the wet mount although widely used, easy, rapid and inexpensive yet, it has low sensitivity. So, negative cases should be repeated by either culture or PCR. El-Okbi et al. (2004) examined vaginal washouts from symptomatic and asymptomatic patients by wet mount examination and culture on modified TYM medium. They found that of 320 cases, 10 were positive for $T$. vaginalis trophozoites by wet mount examination and culture modified TYM medium proved very satisfactory for isolation as well as maintenance of the 10 isolates. They found that antigenic differentiation of the $10 \mathrm{~T}$. vaginalis isolates via SDS-PAGE showed 34 bands using 10\% resolution gel, with molecular weight ranged from 12 to $189 \mathrm{KDa}$. Most of the bands were common among several isolates while Isolate 2 differed than other isolates with two characteristic bands; one at $136 \mathrm{KDa}$ and the other at $25 \mathrm{KDa}$, and isolates $4 \& 8$ had characteristic bands at $163 \mathrm{KDa} \& 189 \mathrm{KDa}$ respectively.

El-Gayar and Rashwan (2007) concluded that early detection and treatment of symptomatic and asymptomatic trichomoniasis minimized the risks of cervical neoplasia. ElSherbini et al. (2010) treated patients with metronidazole refractory vaginal trichomoniasis with natural plant extract purified from pomegranate (Roman) was in-vitro investiga ted for its efficacy against $T$. vaginalis on Diamond media. Infected women $(18 / 20)$ who accepted to be treated with $P$. granatum juice were completely cured and followedup for two months. The anti-trichomoniasis vaginalis activity of $P$. granatum extract (invitro \& in-vivo) gave very promising results. El-Sherbini and El Sherbine (2011) found that the anti- $T$. vaginalis activity of Commiphora molmol gave promising results as a new anti-Trichomonas agents.

El Sibaei et al. (2012) found that T. tenax 
as a pathogen was clearly implicated in various pathological processes that raised outside the mouth boundaries. They studied different isolates of T. tenax from individuals having oral infections and concluded that the high frequency of $T$. tenax detected $(28.6 \%)$ along with the variability in protein profiling and proteolytic activity of the isolates supports the possible pathogenicity of T. tenax and clarifies a conclusion that different strains with possibility of variable pathogenic potential may exist. Kamal et al. (2018) reported that pregnant women lived in rural area with a low socioeconomic and primary educational levels must be screened for trichomoniasis to reduce the incidence of preterm delivery and low birth weight. Saleh et al. (2021) concluded that chronic T. vaginalis infection may be associated with prostate cancer, but it did not seem that this STI aggravated the cancer status.

\section{Recommendations}

- Health illustrative education is a must.

- Asymptomatic carriage can occur for prolonged periods of time, thus it is not necessarily possible to ascertain when or from whom the infection was acquired.

- None of clinical features of trichomonas vaginitis is sufficiently sensitive or specific to allow a diagnosis based upon signs and symptoms alone. Presence of motile trichomonads on wet mount is diagnostic of infection, but only 50 to $70 \%$ of culture- cases. Culture is a must in patients with suggestive clinical findings or when microscopy is unavailable or unreliable. Several rapid antigen tests are now available for diagnosis of trichomonas vaginalis and can be used as an alternative to culture.

- Treatment to relieve symptoms and prevent sexual transmission. A single oral dose of a 5-nitroimidazole drug was recommended (metronidazole, tinidazole) for non-pregnant women (Grade 1A).

- Single dose is more convenient as multiple dose therapy. Oral administration is more effective than vaginal administration.
Sexual partners evaluated for trichomoniasis and sexually transmitted infections rather than empirically treated (Grade 2B).

- Not treating asymptomatic trichomoniasis in pregnant women given the potentially increased of preterm birth risk associated with antibiotic therapy (Grade 2B), and treatment of symptomatic women (Grade 2C).

- Increasing the dose and duration of medication in refractory cases (Grade 2C).

- Patients should be instructed to avoid intercourse until they and their partners have completed treatment and are asymptomatic, which generally takes about a week.

\section{References}

Adu-Sarkodie, Y, Opoku, BK, Danso, KA, Weiss, HA, Mabey, D, 2004: Comparison of latex agglutination, wet preparation, and culture for the detection of Trichomonas vaginalis. Sex. Transm. Infect. 80, 3:201-3.

Anderson, MR, Klink, K, Cohrssen, A, 2004: Evaluation of vaginal complaints. JAMA 291: 1368-72.

Andrea, SB, Chapin, KC, 2011: Comparison of Aptima Trichomonas vaginalis transcriptionmediated amplification assay and BD affirm VPIII for detection of $T$. vaginalis in symptomatic women: performance parameters and epidemiological implications. J. Clin. Microbiol. 49:866-9.

Bhesania, AH, Narayankhedkar, A, 2016: Trichomoniasis: A review. Int. J. Curr. Microbiol. Appl. Sci. 5:731-41.

Carey, JC, Klebanoff, MA, 2005: Is a change in the vaginal flora associated with an increased risk of preterm birth? Am. J. Obstet. Gynecol. 192:1341-4.

Caro-Patón, T, Carvajal, A, Martin de Diego, I, et al, 1997: Is metronidazole teratogenic? A metaanalysis. Br. J. Clin. Pharmacol. 44:179-82.

Carter, JE, Whithaus, KC, 2008: Neonatal respiratory tract involvement by Trichomonas vaginalis: A case report and review of the literature. Am. J. Trop. Med. Hyg. 78:17-20.

CDC, 2006a: Expedited partner therapy in the management of sexually transmitted diseases: US Department of Health and Human Services; Atlanta, GA.

CDC, 2006b: Sexually Transmitted Diseases Treatment Guidelines. MMWR Recomm. Rep. 55(RR-11):1-9. 
CDC, 2010a: Workowski, KA, Berman, S, Sexually transmitted diseases treatment guidelines, MMWR Recomm. Rep. 59:1-8.

CDC, 2010b: Sexually transmitted diseases treatment guidelines. MMWR Recomm. Rep.59,R12 CDC, 2012: National Center for HIV/AIDS, Viral Hepatitis, \& TB; Division of STD Prevention: Trichomoniasis - CDC Fact Sheet.

CDC, 2015: STD Treatment Guidelines - Trichomoniasis. MMWR Recomm. Rep.

CDC, 2017: Trichomoniasis Treatment and Care Chapin, K, Andrea, S, 2011: APTIMA ${ }^{\circledR}$ Trichomonas vaginalis, a transcription-mediated amplification assay for detection of Trichomonas vaginalis in urogenital specimens. Expert. Rev. Mol. Diagn. 11:679-82.

Cotch, MF, Pastorek, JG $2^{\text {nd }}$, Nugent, RP, et al, 1997: Trichomonas vaginalis associated with low birth weight and preterm delivery: The Vaginal Infections and Prematurity Study Group. Sex. Transm. Dis. 24:353-8.

Cudmore, SL, Delgaty, KL, Hayward-McClelland, SF, et al, 2004: Treatment of infections caused by metronidazole-resistant Trichomonas vaginalis. Clin. Microbiol. Rev.17:783-8.

Dan, M, Sobel, JD, 2007: Failure of nitazoxanide to cure trichomoniasis in three women. Sex. Transm. Dis. 34:813-6.

Dessì, D, Margarita, V, Cocco, AR, Marongiu, A, Fiori, PL, et al, 2019: Trichomonas vaginalis and Mycoplasma hominis: New tales of two old friends. Parasitology 146, 9:1150-5

d'Oro, LC, Parazzini, F, Naldi, L, La Vecchia, C, 1994: Barrier methods of contraception, spermicides, and sexually transmitted diseases: a review. Genitourin. Med. 70:410-4.

duBouchet, L, Spence, MR, Rein, MF, et al, 1997: Multicenter comparison of clotrimazole vaginal tablets, oral metronidazole, and vaginal suppositories containing sulfanilamide, aminacrine hydrochloride, and allantoin in the treatment of symptomatic trichomoniasis. Sex. Transm. Dis. 24:156-60.

El-Ganayni, GA, Youssef, MM, el-Shazly, AM, 1992: A preliminary study on vaginal trichomonal antigens using CIEP and modified ELISA. J. Egypt. Soc. Parasitol. 22, 1:145-51.

El-Gayar, EK, Rashwan, MF, 2007: Cervical intraepithelial neoplasia (CIN) and Trichomonas vaginalis infection as revealed by polymerase chain reaction. J. Egypt. Soc. Parasitol. 37, 2: 623-30.

El-Okbi, LM, Arafa, M, Salama, MS, Abou-
El-Seoud, SM, Mohamad, AA, et al, 2004: Growth patterns and antigenic analysis of Egyptian Trichomonas vaginalis isolates. J. Egypt. Soc. Parasitol. 34, 3:841-55

El-Shazly, AM, El-Naggar, HM, Soliman, M, El-Negeri, M, El-Nemr, HE, et al, 2001: A study on trichomoniasis vaginalis and female infertility. J. Egypt. Soc. Parasitol. 31:545-53.

El-Sherbini, GM, Ibrahim, KM, El Sherbiny, ET, Abdel-Hady, NM, Morsy, TA, 2010: Efficacy of Punica granatum extract on in-vitro and in-vivo control of Trichomonas vaginalis. J. Egypt. Soc. Parasitol. 40, 1:229-44.

El-Sherbiny, GM, el Sherbiny, ET, 2011: The effect of Commiphora molmol (Myrrh) in treatment of Trichomoniasis vaginalis infection. Iran Red Crescent Med. J. 13, 7:480-6.

El Seoud, SF, Abbas, MM, Habib, FS, 1988: Study of trichomoniasis among Egyptian male patients. J. Egypt. Soc. Parasitol. 28, 1:263-70.

El Sibaei, MM, Abdel-Fattah, NS, Ahmed, S A, Abou-Seri, HM, 2012: Growth kinetics, antigen profiling, and proteinase activity of Egyptian Trichomonas tenax isolates derived from patients having oral infections. Exp. Parasitol. 130, 4:416-22

Evaldson, GR, Lindgren, S, Nord, CE, Rane, AT, 1985: Tinidazole milk excretion and pharmaco-kinetics in lactating women. Br. J. Clin. Pharmacol. 19, 4:503-7.

Forna, F, Gülmezoglu, AM, 2003: Interventions for treating trichomoniasis in women. Cochrane Database Syst. Rev.:CD000218.

Francis, SC, Kent, CK, Klausner, JD, et al, 2008: Prevalence of rectal Trichomonas vaginalis and Mycoplasma genitalium in male patients at the San Francisco STD clinic, 2005-2006. Sex. Transm. Dis 35:797-800.

Gatski, M, Martin, DH, Levison, J, et al, 2011: The influence of bacterial vaginosis on the response to Trichomonas vaginalis treatment among HIV-infected women. Sex. Transm. Infect. 87:205-8.

Ginocchio, CC, Chapin, K, Smith, JS, et al, 2012: Prevalence of Trichomonas vaginalis and coinfection with Chlamydia trachomatis and Neisseria gonorrhoeae in the United States as determined by Aptima Trichomonas vaginalis nucleic acid amplification assay. J. Clin. Microbiol. 50:2601-6.

Grodstein, F, Goldman, MB, Cramer, DW, 1993: Relation of tubal infertility to history of sexually transmitted diseases. Am. J. Epidemiol. 
137:577-80.

Gray, RH, Kigozi, G, Serwadda, D, et al, 2009: The effects of male circumcision on female partners' genital tract symptoms and vaginal infections in a randomized trial in Rakai, Uganda. Am. J. Obstet. Gynecol. 200:e41-7.

Hager, WD, Brown, ST, Kraus, SJ, et al, 1980: Metronidazole for vaginal trichomoniasis: Seven-day vs single-dose regimens. JAMA 244: 1219-23.

Hammill, HA, 1989: Trichomonas vaginalis. Obstet. Gynecol. Clin. North Am. 16:531-6.

Heine, P, McGregor, JA, 1993: Trichomonas vaginalis: A reemerging pathogen. Clin. Obstet. Gynecol. 36:137-40.

Helms, DJ, Mosure, DJ, Secor, WE, Workowski, KA, 2008: Management of Trichomonas vaginalis in women with suspected metronidazole hypersensitivity. Am. J. Obstet. Gynecol. 198: 370.e1.

Henien, M, Nyirjesy, P, Smith, K, 2019: Metronidazole-resistant trichomoniasis: Beneficial pharmacodynamic relationship with high-dose oral tinidazole and vaginal paromomycin combination therapy. Sex. Transm. Dis. 46, 1:e1-2.

Hesseltine, H, 1942: Experimental human vaginal trichomoniasis. J. Infect. Dis. 71:127-30.

Hoffman, DJ, Brown GD, Wirth FH, et al, 2003: Urinary tract infection with Trichomonas vaginalis in a premature newborn infant and the development of chronic lung disease. J. Perinatol. 23:59-64.

Huppert, JS, Batteiger, BE, Braslins, P, et al, 2005: Use of an immunochromatographic assay for rapid detection of Trichomonas vaginalis in vaginal specimens. J. Clin. Microbiol. 43:68492.

Huppert, JS, Mortensen, JE, Reed, JL, et al, 2007: Rapid antigen testing compares favorably with transcription-mediated amplification assay for detection of Trichomonas vaginalis in for the Trichomonas vaginalis in young women. Clin. Infect. Dis. 45:194-8.

Kamal, AM, Ahmed, AK, Mowafy, NME, Shawki, HE, Sanad, AS, et al, 2018: incidence of antenatal trichomoniasis and evaluation of its role as a cause of preterm birth in pregnant women referring to Minia University Hospital, Egypt. Iran J. Parasitol. 13, 1:58-66.

Kim, JH, Han, IH, Kim, SS, Park, SJ, Min, DY, et al, 2017: Interaction between Trichomonas vaginalis and the prostate epithelium. Korean J. Parasitol. 55, 2:213-8.
Kirkcaldy, RD, Augostini, P, Asbel, LE, et al, 2012: Trichomonas vaginalis antimicrobial drug resistance in 6 US cities, STD Surveillance Network, 2009-2010. Emerg. Infect. Dis. 18:939-42. Kissinger, P, Mena, L, Levison, J, et al, 2010: A randomized treatment trial: Single versus 7day dose of metronidazole for the treatment of Trichomonas vaginalis among HIV-infected women. J. Acquir. Immune Defic. Syndr. 55:565-9. Kissinger, P, Schmidt, N, Mohammed, H, et al, 2006: Patient-delivered partner treatment for Trichomonas vaginalis infection: A randomized controlled trial. Sex. Transm. Dis. 33:445-8.

Klebanoff, MA, Carey, JC, Hauth, JC, et al, 2001: Failure of metronidazole to prevent preterm delivery among pregnant women with asymptomatic Trichomonas vaginalis infection. N. Engl. J. Med. 345:487-90.

Klebanoff, MA, Carey, JC, Hauth, JC, Hillier, SL, Nugent, RP, et al, 2001: Failure of metronidazole to prevent preterm delivery among pregnant women with asymptomatic Trichomonas vaginalis infection. New Engl. J. Med. 345, 7: 487-93.

Krieger, JN, Tam, MR, Stevens, CE, et al, 1988: Diagnosis of trichomoniasis: Comparison of conventional wet-mount examination with cytologic studies, cultures, and monoclonal antibody staining of direct specimens. JAMA 259: 1223-8.

Lactmed, 2010: Available at file://toxnet.nlm.ni h.gov/cgi-bin/sis/search/f?./temp/ R2NQWq:2.

Laga, M, Manoka, A, Kivuvu, M, et al, 1993: Non-ulcerative sexually transmitted diseases as risk factors for HIV-1 transmission in women: results from a cohort study. AIDS7:95-8.

Langston, ME, Bhalla, A, Alderete, JF, Nevin, RL, Pakpahan, R, et al, 2019: Trichomonas vagin-alis infection and prostate-specific antigen concentration: Insights into prostate involvement and prost-ate disease risk. Prostate 79, 14:1622-8 Lara-Torre, E, Pinkerton, JS, 2003: Accuracy of detection of trichomonas vaginalis organisms on a liquid-based papanicolaou smear. Am. J. Obstet. Gynecol.188:354-8.

Lossick, JG, 1990: Treatment of sexually transmitted vaginosis/vaginitis. Rev. Infect. Dis.12, 6: S665-9.

Loveless, M, Myint, O, 2018: Vulvovaginitispresentation of more common problems in pediatric and adolescent gynecology. Best Pract. Res. Clin. Obstet. Gynaecol. 48:14-27.

Mahmoud, A, Sherif, NA, Abdella, R, El-Gen- 
edy, AR, El Kateb, AY, et al, 2015: Prevalence of Trichomonas vaginalis infection among Egyptian women using culture and Latex agglutination: Cross-sectional study. BMC Women Hlth. 15:7. doi: 10.1186/s12905-015-0169-2.

Manorama, HT, Shenoy, DR, 1978: Single-dose oral treatment of vaginal trichomoniasis with tinidazole and metronidazole. J. Int. Med. 6:4650.

McClelland, RS, Sangare, L, Hassan, WM, et al, 2007: Infection with Trichomonas vaginalis increases the risk of HIV-1 acquisition. J. Infect. Dis. 195:698-702.

McGregor, JA, French, JI, Parker, R, Draper, D, Patterson, E, et al, 1995: Prevention of premature birth by screening and treatment for common genital tract infections: results of a prospective con-trolled evaluation. Am. J. Obstet. Gynecol. 173, 1:157-67

Michalowski, R, 1981: Trichomonal balanoposthitis: Report of 16 cases. Ann. Dermatol. Venereol. 108, 10:731-8.

Mielczarek, E, Blaszkowska, J, 2016: Trichomonas vaginalis: pathogenicity and potential role in human reproductive failure. Infection 44, 4:447-58

Moodley, P, Wilkinson, D, Connolly, C, et al, 2002: Trichomonas vaginalis is associated with pelvic inflammatory disease in women infected with human immunodeficiency virus. Clin. Infect. Dis. 34:519-22.

Morsy, TA, Hosni, MA, el-Masry, G, 1984: Clinical and laboratory studies on vaginal trichomoniasis in Egypt. J. Egypt. Soc. Parasitol. 14, 2:329-34

Muzny, CA, Schwebke, JR, 2013: The clinical spectrum of Trichomonas vaginalis infection \& challenges to management. Sex. Transm. Infect. $89,6: 423-5$

Negm, AY, el-Haleem, DA, 2004: Detection of trichomoniasis in vaginal specimens by both conventional and modern molecular tools. J. Egypt. Soc. Parasitol. 34, 2:589-600.

Nye, MB, Schwebke, JR, Body, BA, 2009: Comparison of APTIMA Trichomonas vaginalis transcription-mediated amplification to wet mount microscopy, culture, and polymerase chain reaction for diagnosis of trichomoniasis in men and women. Am. J. Obstet. Gynecol. 200, 188: e1.

Nyirjesy, P, Weitz, MV, Gelone, SP, Fekete, T, 1995: Paromomycin for nitroimidazole-resistant trichomonosis. Lancet 346:1110-4.
Okun, N, Gronau, KA, Hannah, ME, 2005: Antibiotics for bacterial vaginosis or Trichomonas vaginalis in pregnancy: A systematic review. Obstet. Gynecol. 105:857-60.

Patel, EU, Gaydos, CA, Packman, ZR, et al, 2018: Prevalence and correlates of Trichomonas vaginalis infection among men and women in the United States. Clin. Infect. Dis. 67, 2:211-7.

Peterman, T, Tian, L, Metcalf, C, et al, 2006: High incidence of new sexually transmitted infections in the year following a sexually transmitted infection: a case for rescreening. Ann. Intern. Med. 145:564-72.

Poppe WA, 2001: Nitroimidazole-resistant vaginal trichomoniasis treated with paromomycin. Eur. J. Obstet. Gynecol. Reprod. Biol. 96:11923.

Rowley, J, Hoorn, S, Korenromp, E, et al, 2019: Chlamydia, gonorrhoea, trichomoniasis and syphilis: Global prevalence and incidence estimates, 2016. Bull. WHO. 97, 8:P548-62.

Saleh, NE, Alhusseiny, SM, El-Zayady, WM, Aboelnaga, EM, El-Beshbishi, WN, et al, 2021: Trichomonas vaginalis serostatus and prostate cancer risk in Egypt: A case-control study. Parasitol. Res. 120, 4:1379-88

Sayed el-Ahl, SA, el-Wakil, HS, Kamel, NM, Mahmoud, MS, 2002: A preliminary study on the relationship between Trichomonas vaginalis and cervical cancer in Egyptian women. J. Egypt. Soc. Parasitol. 32, 1:167-78.

Schwebke, JR, Barrientes, FJ, 2006: Prevalence of Trichomonas vaginalis isolates with resistance to metronidazole and tinidazole. Antimicrob. Agents Chemother. 50:4209-12.

Schwebke, JR, Burgess, D, 2004: Trichomoniasis. Clin. Microbiol. Rev.17:794-9.

Schwebke, JR, Hobbs, MM, Taylor, SN, et al, 2011: Molecular testing for Trichomonas vaginalis in women: results from a prospective U.S. clinical trial. J. Clin. Microbiol. 49:4106-9.

Schwebke, JR, Lensing, S, Sobel, J, 2013: Intravaginal metronidazole/miconazole for the treatment of vaginal trichomoniasis. Sex. Transm. Dis. 40:710-4.

Seña, AC, Miller, WC, Hobbs, MM, et al, 2007: Trichomonas vaginalis infection in male sexual partners: implications for diagnosis, treatment, and prevention. Clin. Infect. Dis. 44:13-8. Shui, IM, Kolb, S, Hanson, C, Sutcliffe, S, Rider, JR, et al, 2016: Trichomonas vaginal$i s$ infection and risk of advanced prostate cancer. Prostate 76, 7:620-3. 
Sivaraj, V, Ahamed, A, Artykov, R, MenonJohansson, A, 2021: Epididymitis and its aetiologies in a central London sexual health clinic. Int. J. STD AIDS 32, 1:96-9.

Smith, LM, Wang, M, Zangwill, K, Yeh, S, 2002: Trichomonas vaginalis infection in a premature newborn. J. Perinatol. 22:502-6.

Sobel, JD, Nagappan, V, Nyirjesy, P, 1999: Metronidazole-resistant vaginal trichomoniasis: An emerging problem. N. Engl. J. Med. 341: 292-6.

Sobel, JD, Nyirjesy, P, Brown, W, 2001: Tinidazole therapy for metronidazole-resistant vaginal trichomoniasis. Clin. Infect. Dis. 33:1341-4. Soper, D, 2004: Trichomoniasis: Under control or undercontrolled? Am. J. Obstet. Gynecol. 190 :281-6.

Soper, DE, Bump, RC, Hurt, WG, 1990: Bacterial vaginosis and trichomoniasis vaginitis are risk factors for cuff cellulitis after abdominal hysterectomy. Am. J. Obstet. Gynecol. 163:101620.

Sorvillo, F, Kerndt, P, 1998: Trichomonas vaginalis and amplification of HIV-1 transmission.

Lancet 351:213-2.

Stoner, KA, Rabe, LK, Meyn, LA, Hillier, SL, 2013: Survival of Trichomonas vaginalis in wet preparation and on wet mount. Sex. Transm. Infect. 89, 6:485-8.

Stukus, KS, Buckingham, D, Cohen, DM, 2019: Increasing Trichomonas vaginalis testing for high-risk adolescents a pediatric emergency department. Pediatr. Qual. Saf. 4, 2:e140.

Subramanian, C, Sobel, JD, 2011: A case of high-level metronidazole-resistant trichomoniasis in pregnancy successfully treated. J. Low Genit. Tract Dis. 15:248-51.

Sutton, M, Sternberg, M, Koumans, EH, et al, 2007: The prevalence of Trichomonas vaginalis infection among reproductive-age women in the United States, 2001-2004. Clin. Infect. Dis. 45: 1319-22.

Tawfeek, GM, Oteifa, NM, el-Gozamy, BR, 2003: Evaluation of an IgG cystatin capture enzyme-linked immunosorbent assay for the detection of anti-cysteine proteinase antibodies in asymptomatic trichomoniasis patients. J. Egypt. Soc. Parasitol. 33, 1:67-83.

Temesvári, P, Kerekes, A, Tege, A, Szarka, K, 2002: Demonstration of Trichomonas vaginalis in tracheal aspirates in infants with early respira- tory failure. J. Matern. Fetal Neonatal Med. 11: 347-50.

Thin, RN, Symonds, MA, Booker, R, et al, 1979: Double-blind comparison of a single dose and a five-day course of metronidazole in the treatment of trichomoniasis. Br. J. Vener. Dis. 55:354-8.

Tidwell, BH, Lushbaugh, WB, Laughlin, MD, et al, 1994: A double-blind placebo-controlled trial of single-dose intravaginal versus single-dose oral metronidazole in the treatment of Trichomonal vaginitis. J. Infect. Dis. 170:242-8.

Tsai, CS, Shepherd, BE, Vermund, SH, 2009: Use of nucleic acid amplification testing for diagnosis of anorectal sexually transmitted infections. J. Clin. Microbiol. 50:2005-8.

Van Der Pol, B, Kraft, CS, Williams, JA, 2006: Use of an adaptation of a commercially available PCR assay aimed at diagnosis of chlamydia and gonorrhea to detect Trichomonas vaginalis in urogenital specimens. J. Clin. Microbiol. 44:366-70.

Vos, T, Barber, RM, Bell, B, Bertozzi-Villa, A, Stan Biryukov, S, et al, 2015: GBD Study 2013 Collaborators, Global, regional, and national incidence, prevalence, and years lived with disability for 301 acute and chronic diseases and injuries in 188 countries, 1990-2013: A systematic analysis for the Global Burden of Disease Study 2013. Lancet 386, 9995:743-800.

Vos, T, Flaxman, AD, Naghavi, M, Lozano, R, Michaud, C, et al, 2012: Years lived with disability (YLDs) for 1160 sequelae of 289 diseases and injuries 1990-2010: a systematic analysis for the Global Burden of Disease Study 2010. Lancet 380, 9859:2163-96.

Wiese, W, Patel, SR, Patel, SC, et al, 2000: A meta-analysis of the Papanicolaou smear and wet mount for the diagnosis of vaginal trichomoniasis. Am. J. Med. 108:301-6.

Wølner-Hanssen, P, Krieger, JN, Stevens, C E, et al, 1989: Clinical manifestations of vaginal trichomoniasis. JAMA 261:571.

Workowski, KA, Bolan, GA, 2015: Sexually transmitted diseases treatment guidelines. MMWR. Re-commendations and Reports 64, RR-03: $1-137$.

Zhang, ZF, Begg, CB, 1994: Is Trichomonas vaginalis a cause of cervical neoplasia? results from a combined analysis of 24 studies. Int. J. Epidemiol. 23:682-8. 